ISSN 0103-5150

Fisioter. Mov., Curitiba, v. 26, n. 2, p. página 321-327, abr./jun. 2013

Licenciado sob uma Licença Creative Commons

\title{
Efeitos de diferentes modalidades de treinamento físico e do hábito de caminhar sobre o equilíbrio funcional de idosos
}

\author{
Effects of different methods of physical training and the \\ habit of walking on functional balance of elderly
}

\author{
Carla Helrigle $^{[a]}$, Lucila Pessuti Ferri ${ }^{[b]}$, Carolina Pereira de Oliveira Netta ${ }^{[c]}$, Jessika Barbosa Belem ${ }^{[\mathrm{d}]}$, \\ Tais Malysz ${ }^{[e]}$ \\ [a] Fisioterapeuta, especialista em Prescrição de Exercício Físico pela Universidade Federal de Goiás (UFG), Jataí, GO - Brasil, \\ e-mail: carlahelrigle@hotmail.com \\ [b] Enfermeira, especialista em Saúde Pública, mestranda em Saúde Coletiva pela Universidade Federal de Goiás, (UFG), \\ Goiânia, GO - Brasil, e-mail: cilapessuti@bol.com.br \\ [c] Educadora Física pela Universidade Federal de Goiás (UFG), Jataí, GO - Brasil, e-mail: carol-ipij@gmail.com \\ [d] Educadora Física pela Universidade Federal de Goiás (UFG), Jataí, GO - Brasil, e-mail: jesskbb@yahoo.com.br \\ [e] Fisioterapeuta, doutora em Neurociências pela Universidade Federal do Rio Grande do Sul (UFRGS), docente da \\ Universidade Federal de Goiás (UFG), Jataí, GO - Brasil, e-mail: taismalysz@yahoo.com.br
}

\section{Resumo}

Introdução: As alterações do equilíbrio corporal estão entre as queixas mais comuns da população idosa e constituem um problema médico de grande relevância. Objetivo: Comparar a influência da prática de diferentes modalidades de treinamento físico e do hábito de caminhar sobre o equilíbrio funcional de idosos residentes em Jataí, Goiás. Materiais e métodos: Fizeram parte desta pesquisa 135 idosos divididos em cinco grupos de acordo com o hábito de caminhar e com a modalidade de treinamento físico praticada: sedentários inativos ( $\mathrm{n}=39)$, sedentários ativos $(\mathrm{n}=37)$, hidroginástica $(\mathrm{n}=25)$, musculação $(\mathrm{n}=10)$ e caminhada $(\mathrm{n}=$ 24). 0 equilíbrio funcional foi avaliado a partir do resultado atingido na Escala de Equilíbrio de Berg (EEB). Os dados foram expressos em valores de média e desvio do erro padrão e comparados entre si por meio da Análise de Variância One-Way seguida do teste post-hoc Tukey $(\mathrm{p}<0,05)$, utilizando-se o software SPSS 12.0. Resultados: Os idosos sedentários inativos e ativos atingiram valores de 48,89 $\pm 0,87$ e 52,45 $\pm 0,47$, respectivamente na EEB. Os idosos treinados praticantes de hidroginástica obtiveram escore de 54,04 $\pm 0,33$, os praticantes de musculação $53 \pm 0,52$ e os praticantes de caminhada de 53,45 $\pm 0,51$. A análise estatística 
mostrou que tanto os indivíduos sedentários ativos quanto os idosos treinados obtiveram maior valor atingido na EEB em comparação com os sedentários inativos $(\mathrm{p}<0,05)$. Não foram observadas diferenças estatísticas entre os diferentes grupos de idosos treinados e nem destes com o grupo de idosos sedentários ativos. Conclusão: Tanto o hábito de caminhar quanto a prática regular da caminhada, da musculação e da hidroginástica por mais de seis meses, aumentam o equilíbrio funcional dos idosos.

Palavras-chave: Idosos. Treinamento. Equilíbrio.

\begin{abstract}
Introduction: The balance disorders are among the most common complaints of the elderly population and constitute a medical problem of high relevance. Objective: To compare the influence of the practice of various methods of physical training and habit of walking on the balance of elderly residents in Jataí, Goias State, Brazil. Materials and methods: One hundred thirty-five elderly were divided into five groups according to the habit of walking and the sport practiced: sedentary $(n=39)$, active sedentary $(n=37)$, hidrogymnastics $(n=25)$, weightlifting $(n=10)$ and walking training $(n=24)$. Functional balance was assessed by the results achieved in the Berg Balance Scale. Data were expressed as mean and standard error deviation and compared by analysis of variance One-Way followed by Tukey's post hoc test $(p<0.05)$ using software SPSS 12.0 . Results: The sedentary and active sedentary groups assets reached values of $48.89 \pm 0.87$ and $52.45 \pm 0.47$, respectively in the scale. The elderly trained practitioners of hidrogymnastics had a score of $54.04 \pm 0.33$, the bodybuilders $53 \pm 0.52$ and walking training $53.45 \pm 0.51$. Statistical analysis showed that both active sedentary and trained groups had higher score on the Berg Scale compared with sedentary group $(p<0.05)$. There were no statistical differences between different groups of trained elderly and even those trained with the group of active sedentary group. Conclusion: Both the habit of walking as a regular practice of and hydrogymnastics, weightlifting and walking training for more than six months, increase the functional balance of the elderly.
\end{abstract}

Keywords: Elderly. Training. Balance.

\section{Introdução}

A população geriátrica apresenta um crescente aumento dos distúrbios das funções sensoriais, da integração das informações periféricas centrais, bem como a senescência dos sistemas neuromusculares e da função esquelética (1). Os idosos também são mais propensos às doenças que alteram diretamente essas funções como o diabetes, a aterosclerose, doenças reumatológicas, acidente vascular cerebral, depressão, entre outras (2). Além disso, a perda progressiva das aptidões funcionais do indivíduo idoso aumenta a propensão ao sedentarismo (3), que pode prejudicar a qualidade de vida do idoso e colocar em maior vulnerabilidade a sua saúde.

As alterações do equilíbrio corporal estão entre as queixas mais comuns da população idosa e constituem um problema médico de grande relevância. Estima-se que a prevalência de queixas de equilíbrio chegue a $85 \%$ da população acima de 65 anos (4) e que as quedas aumentem em idosos com idade mais avançada, atingindo $30 \%$ daqueles entre 65 e 74 anos e $40 \%$ entre aqueles com 75 anos ou mais (5).

Sabe-se que o treinamento físico de uma forma geral aumenta o equilíbrio de idosos (6); a hidroginástica (7), os exercícios específicos para o sistema vestibular (8), a cinesioterapia (9) e a caminhada (10), por exemplo, contribuem para o aprendizado motor, para a melhora do equilíbrio e para a diminuição na possibilidade de queda em idosos.

Embora os idosos que não realizam treinamento físico regularmente sejam considerados sedentários, sabe-se que existem idosos sedentários mais ativos que outros. Muitos possuem o hábito de permanecer mais tempo dentro de casa e outros, mais ativos, possuem o hábito de caminhar fora de casa para realizar diferentes atividades, como a de ir ao supermercado, visitar amigos etc. Segundo Matsudo (11), para envelhecer com saúde e qualidade, tão importante quanto estimular a prática regular da atividade física é a adoção de um estilo de vida mais ativo. 
Considerando a carência de estudos comparativos entre diferentes formas de atividades físicas sobre o equilíbrio de idosos, o objetivo deste trabalho foi o de comparar a influência da prática de diferentes modalidades de treinamento físico (caminhada, musculação e hidroginástica) e do hábito de caminhar fora de casa sobre o equilíbrio funcional e risco de quedas de idosos residentes em Jataí, Goiás.

\section{Materiais e métodos}

Fizeram parte desta pesquisa 135 idosos de ambos os sexos com idade entre 60 e 82 anos e que concordaram em participar da pesquisa, assinando o Termo de Consentimento Livre e Esclarecido. Este estudo foi aprovado pelo Comitê de Ética em Pesquisa da Universidade Federal de Goiás (185/2009). Todos os idosos apresentaram independência na marcha e nas atividades funcionais da vida diária (banhar-se, alimentar-se e vestir-se) e cognitivo preservado para entendimento de ordens simples.

Inicialmente os idosos foram divididos em dois principais grupos, divididos em razão dos hábitos de prática de treinamento físico regular: grupo sedentário $(n=76)$ e grupo treinado $(n=59)$. Foram considerados sedentários os indivíduos que não praticaram treinamento físico regular nos seis meses prévios ao estudo, e treinados os que praticaram regularmente, nos últimos seis meses, pelo menos duas sessões semanais de atividade física estruturada, com duração de aproximadamente 50 minutos e dirigidas para vários componentes de aptidão física (treino de resistência aeróbia, força muscular e flexibilidade).

De acordo com o hábito de caminhar fora de casa, os idosos sedentários foram distribuídos em dois subgrupos: sedentários inativos $(n=39)$ e sedentários ativos ( $\mathrm{n}=37)$. Os idosos ativos tinham o hábito de caminhar fora de casa por mais de quatro quarteirões, pelo menos três vezes semanais, nos seis meses prévios ao estudo.

Dentro do grupo treinado os idosos foram distribuídos em subgrupos de acordo com a modalidade de praticada: caminhada $(n=24)$, musculação $(n=10)$ e hidroginástica $(\mathrm{n}=25)$. Os participantes desta pesquisa foram recrutados na Policlínica Municipal de Jataí, academias da cidade, Grupo da Hidroginástica do Colégio Bom Conselho e Escola de Natação Mundo Aquático do município de Jataí (GO).
O equilíbrio funcional e risco de quedas foram avaliados por meio dos resultados da Escala de Equilíbrio de Berg (EEB) adaptada para a versão brasileira (12); essa escala leva em conta os efeitos das condições ambientais sobre a função e é composta por 14 testes que avaliam a habilidade do indivíduo de se sentar, ficar em pé, alcançar, girar em volta de si mesmo, olhar por cima de seus ombros, ficar sobre o apoio unipodal e transpor degraus.

A Escala de Equilíbrio de Berg apresenta pontuação que pode variar de 0 a 56; quanto maior o valor atingido, maior é o equilíbrio funcional e menor é o risco de quedas. 0 escore de 45 pontos permite separar indivíduos com risco baixo e moderado de quedas (13) e o índice igual ou menor que 36 está associado a $100 \%$ de risco de quedas (14). Este instrumento apresenta excelente confiabilidade $(0,96)$ e moderada/boa correlação com outros instrumentos de avaliação funcional do equilíbrio como: Escala de Mobilidade de Barthel, 0,67; Teste Up and Go, 0,76; Escala de Equilíbrio de Tinetti, 0.91 (15).

Os dados da EEB foram expressos em valores de média e desvio do erro padrão e comparados entre si por meio da Análise de Variância One-Way (ANOVA) seguida do teste post-hoc Tukey, com significância de $\mathrm{p}<0,05$, utilizando-se o software SPSS 12.0.

\section{Resultados}

A maioria dos indivíduos avaliados foi do sexo feminino $(\mathrm{n}=113)$. Os sedentários inativos (34 mulheres e 5 homens) e os sedentários ativos (28 mulheres e 9 homens) apresentaram média de idade equivalente 70,07 $\pm 0,91$ e 69,48 $\pm 0,94$ anos, respectivamente. Dentre os grupos treinados, os idosos praticantes de hidroginástica (25 mulheres), musculação (6 mulheres e 4 homens) e caminhada (20 mulheres e 4 homens) apresentaram média de idade equivalente a $66,84 \pm 1,17,65,33 \pm 1,08$ e $67,7 \pm 1,24$ anos, respectivamente. 0 tempo de treinamento físico realizado previamente à avaliação do equilíbrio foi igual a 34,08 $\pm 8,3$ meses para os praticantes de hidroginástica, 29,33 $\pm 1,65$ meses para os praticantes de musculação e de $29,25 \pm 4,04$ meses para os praticantes de caminhada.

Os resultados mostraram que 125 idosos apresentaram valores da EEB maiores que 45, 8 idosos apresentaram valores menores ou iguais a 45 e somente 2 obtiveram valores menores ou iguais a 36 (Tabela 1 ). 
Em relação às médias dos escores obtidos na EEB, os idosos sedentários inativos e ativos atingiram valores de 48,89 $\pm 0,87$ e $52,45 \pm 0,47$, respectivamente. Os idosos treinados praticantes de hidroginástica obtiveram escore de $54,04 \pm 0,33$, praticantes de musculação obtiveram $53 \pm 0,52$ e os praticantes de caminhada apresentaram escore de 53,45 \pm 0,51
(Gráfico 1). A análise estatística mostrou que os indivíduos sedentários ativos e os treinados obtiveram maior valor atingido na EEB em comparação com os sedentários inativos $(\mathrm{p}<0,05)$. Não foram observadas diferenças estatísticas entre os diferentes grupos de idosos treinados e nem destes com o grupo de idosos sedentários ativos.

Tabela 1 - Valores da Escala de Equilíbrio de Berg atribuídos aos indivíduos dos diferentes grupos estudados

\begin{tabular}{lccccc}
\hline & $\begin{array}{c}\text { Sedentários } \\
\text { Inativos }(\mathrm{n})\end{array}$ & $\begin{array}{c}\text { Sedentários } \\
\text { Ativos }(\mathrm{n})\end{array}$ & $\begin{array}{c}\text { Praticantes de } \\
\text { hidroginástica (n) }\end{array}$ & $\begin{array}{c}\text { Praticantes de } \\
\text { musculação }(\mathrm{n})\end{array}$ & $\begin{array}{c}\text { Praticantes de } \\
\text { caminhada }(\mathrm{n})\end{array}$ \\
\hline $\mathrm{EEB} \leq 36$ & 2 & 0 & 0 & 0 & 0 \\
$\mathrm{EEB} \leq 45$ & 7 & 1 & 0 & 0 & 0 \\
$\mathrm{EEB}>45$ & 30 & 36 & 25 & 10 & 24 \\
\hline
\end{tabular}

Fonte: Dados da pesquisa.

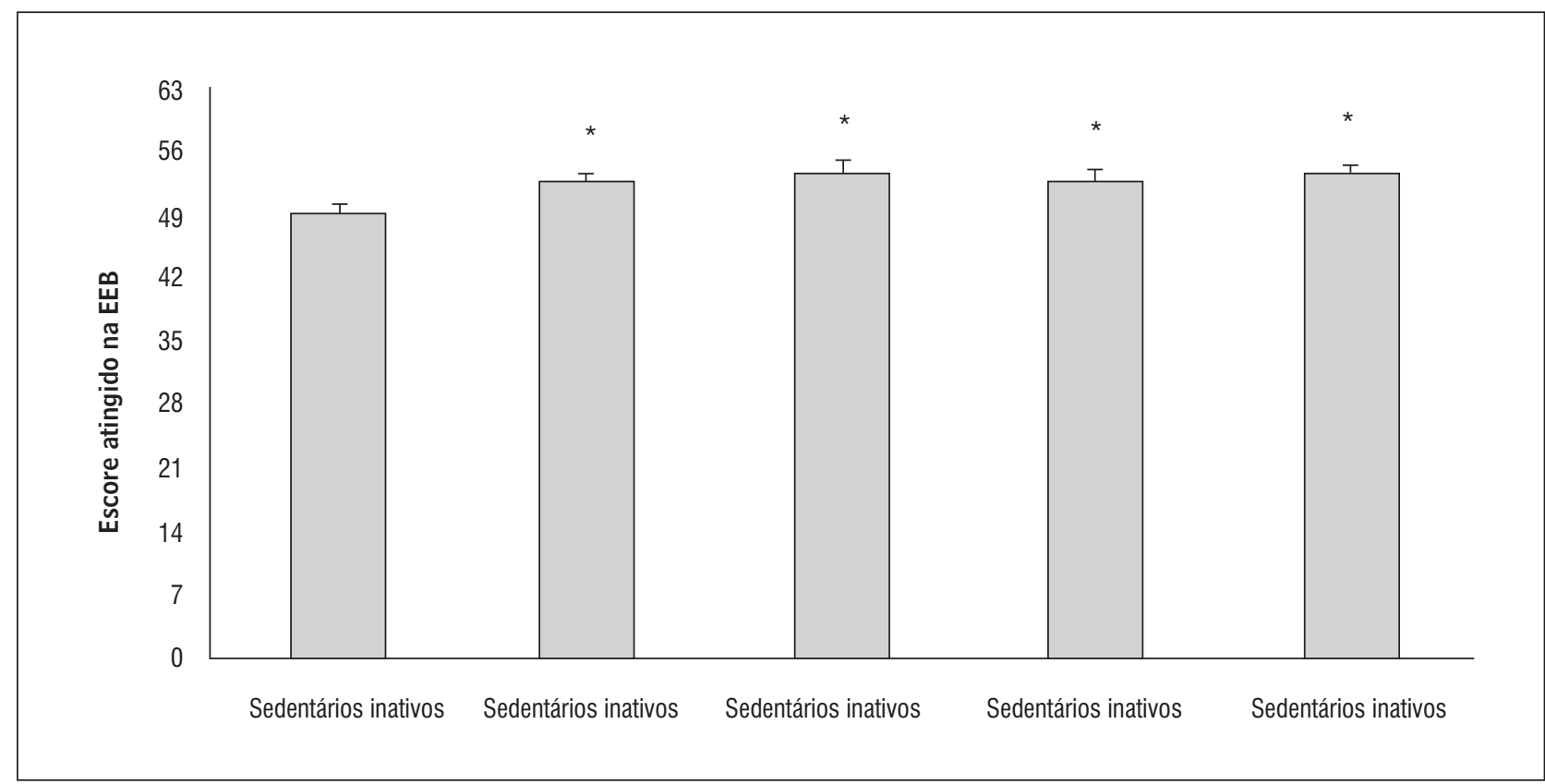

Gráfico 1 - Resultados da Escala de Equilíbrio de Berg dos grupos de idosos treinados e dos grupos de idosos sedentários Nota: ${ }^{*}=$ vs. grupo sedentário inativo $(p<0,05)$.

\section{Discussão}

O equilíbrio, essencial para os indivíduos se movimentarem em seu ambiente e realizarem com êxito as atividades diárias, é caracterizado como a habilidade do sistema nervoso em detectar, de forma antecipada ou momentânea, a instabilidade e gerar respostas coordenadas que tragam de volta para a base de sustentação o centro de gravidade corporal, evitando a queda (16). Os mecanismos necessários para manter o equilíbrio estático e dinâmico incluem uma interação dinâmica entre a captação e transmissão de estímulos sensoriais (visual, proprioceptivo e vestibular) para o sistema nervoso central, integração 
sensório-motora pelo sistema nervoso central e elaboração de respostas motoras a serem conduzidas aos músculos para efetuar os ajustes posturais contínuos (17). Dessa forma, para manter o equilíbrio corporal, torna-se necessário apresentar, além de integridade do sistema sensorial e nervoso (central e periférico), preservação das condições normais do sistema musculoesquelético, as quais resultam em amplitude de movimento e força muscular adequadas para a correta realização dos ajustes posturais (18).

Sabe-se que os distúrbios do equilíbrio estão associados ao processo de envelhecimento e às diversas patologias relacionadas (2). Tais distúrbios são manifestados pelo desequilíbrio corporal, que pode culminar em um evento de queda com possíveis sequelas, aumentando a morbidade nessa população (19). Como decorrência dessas quedas, as fraturas são responsáveis por aproximadamente $70 \%$ das mortes acidentais em pessoas acima de 75 anos (20).

Os efeitos benéficos da prática regular da atividade física no processo de envelhecimento têm sido amplamente descritos (11) e incluem: redução de risco de quedas e de lesões graves destas decorrentes; aumento da força muscular dos membros inferiores e coluna vertebral; e melhora do tempo de reação, da sinergia motora das reações posturais, da velocidade de andar, da mobilidade e da flexibilidade.

De acordo com as classificações de Chiu et al. (13) e Shumway-Cook et al. (14) para as interpretações dos resultados da Escala de Equilíbrio de Berg, os resultados da nossa pesquisa mostraram que todos os idosos treinados apresentaram baixo risco de quedas (valores de EEB > 45). Quanto ao risco de quedas entre os sedentários inativos, embora a maioria tenha apresentado baixo risco, sete idosos apresentaram risco moderado (valores de EEB $\leq 45$ ) e dois idosos apresentaram alto risco (valores de EEB $\leq 36$ ). Entre os idosos sedentários ativos, somente um idoso apresentou moderado risco de quedas e o restante apresentou baixo risco de quedas.

Ao analisar as médias dos escores atingidos na Escala de Equilíbrio de Berg, observamos que todos os grupos de idosos apresentaram baixo risco de quedas. Nossos resultados mostram valores semelhantes aos encontrados por outros autores (13, $14,21)$ que pesquisaram risco de quedas em idosos. Concordando com Bretan et al. (21), acreditamos que uma possível explicação para esses dados é a possibilidade de terem comparecido nos estabelecimentos de saúde e nas academias apenas os idosos mais saudáveis, com doenças mais controladas e com vida diária mais independente.

Entretanto, apesar de todos os grupos terem apresentado baixo risco de quedas, os grupos de indivíduos treinados apresentaram maior pontuação na EEB em comparação com indivíduos sedentários inativos. Da mesma maneira, os indivíduos sedentários ativos apresentaram maior valor atingido nesta escala em comparação aos sedentários inativos. Por meio deste instrumento, nenhuma das modalidades de treinamento físico mostrou promover maior equilíbrio funcional aos idosos, e da mesma forma, também nenhuma delas mostrou ser mais eficaz do que o hábito de caminhar fora de casa.

Diferentes autores concordam que o treinamento físico de idosos no meio aquático $(7,22,23,24)$ e em solo $(25,26,27)$ melhora o equilíbrio corporal estático e dinâmico. Segundo Coelho e Coelho (28), tanto a prática regular da caminhada quanto a prática da hidroginástica por 12 semanas oferecem benefícios para a aptidão física de idosos, como o aumento da capacidade de realização das atividades de vida diária. De forma semelhante, o trabalho realizado por Avelar et al. (24) verificou que exercícios de resistência muscular em membros inferiores, por seis semanas, proporcionam uma melhora significativa no equilíbrio estático e dinâmico de idosos independente do meio em que este é realizado, ou seja dentro ou fora da água.

De uma forma geral o exercício possibilita manutenção da independência funcional na população idosa com o aumento da aptidão cardiorrespiratória, força e resistência muscular e flexibilidade corporal (29), além de diminuir o risco de desenvolvimento de patologias associadas com a idade avançada e a inatividade (30).

\section{Considerações finais}

Tanto o hábito de caminhar fora de casa quanto a prática regular da caminhada, da musculação e da hidroginástica por mais de seis meses, aumentam o equilíbrio funcional e diminuem o risco de quedas em idosos. Não identificamos uma modalidade de treinamento como mais eficaz para essa melhora do equilíbrio funcional. Mostramos, porém, que o hábito de caminhar fora de casa por mais de seis quarteirões por pelo menos três vezes na semana também aumenta o equilíbrio funcional de idosos, reduzindo assim o risco de quedas. 
Acreditamos que, possivelmente, existam diferenças entre os grupos estudados que mostraram ter um bom equilíbrio funcional (treinados/sedentários ativos), ainda que não tenham sido detectadas mediante o instrumento utilizado nesta pesquisa. Sugerimos que futuras pesquisas sejam realizadas para avaliar o equilíbrio de idosos treinados e sedentários ativos por meio de equipamentos biomecânicos, os quais, de forma quantitativa, detectem e analisem as variações no centro de gravidade.

\section{Referências}

1. Davini R, Nunes CV. Alterações no sistema neuromuscular decorrentes do envelhecimento e o papel do exercício físico na manutenção da força muscular em indivíduos idosos. Rev Bras Fisioter. 2003;7:201-7.

2. Simoceli L, Bittar RMS, Bottino MA, Bento RF. Perfil diagnóstico do idoso portador de desequilíbrio corporal: resultados preliminares. Rev Bras Otorrinolaringol. 2003;69:772-77. doi:10.1590/ S0034-72992003000600008.

3. Kallinen M, Markku A. Aging, physical activity and sports injury. An overview of common sports injuries in the ederly. Sports Med. 1995;20:41-52. doi:10.2165/00007256-199520010-00004.

4. Bittar RSM, Pedalini MEB, Sznifer J, Formigoni LG. Reabilitação vestibular: opção terapêutica na síndrome do desequilíbrio do idoso. Gerontologia. 2000;8:9-12.

5. O'Loughlin JL, Robitaille Y, Boivin JF, Suissa S. Incidence of and risk factors for falls and injuries falls among the community-dwelling elderly. Am J Epidemiol. 1993;137:342-54. PMid:8452142.

6. Carvalho J, Pinto J, Mota J. Actividade física, equilíbrio e medo de cair. Um estudo em idosos institucionalizados. Rev Port Cien Desp. 2007;7:225-31.

7. Bruni BM, Granado FB, Prado RA. Avaliação do equilíbrio postural em idosos praticantes de hidroterapia em grupo. O Mundo da Saúde São Paulo. 2008;32:56-63.

8. Ribeiro ASB, Pereira JS. Melhora do equilíbrio e redução da possibilidade de queda em idosas após exercícios de Cawthorne e Cooksey. Rev Bras Otorrinolaringol. 2005;71:38-46. doi:10.1590/ S0034-72992005000100008.
9. Soares MA, Sacchelli T. Efeitos da cinesioterapia no equilíbrio de idosos. Rev Neurocienc. 2008;16:97-100.

10. Salbach NM, Mayo NE, Robichaud-Ekstrand S, Hanley JA, Richards CL, Wood-Dauphinee S. The effect of a task-oriented walking intervention on improving balance self-efficacy poststroke: a randomized, controlled trial. J Am Geriatr Soc. 2005;53:576-82. doi:10.1111/j.1532-5415.2005.53203.x.

11. Matsudo SMM. Envelhecimento, atividade física e saúde. BIS, Bol. Inst. Saúde (Impr.). 2009 [cited May 13th 2011]; 47:76-79 Available from: http://periodicos.ses. sp.bvs.br/scielo.php?script=sci_arttext\&pid=S1518$-18122009000200020 \& \operatorname{lng}=\mathrm{en}$.

12. Miyamoto ST, Lombardi I Jr., Berg KO, Ramos LR, Natour J. Brazilian version of the Berg balance scale. Braz J Med Biol Res. 2004;37:1411-21. doi:10.1590/ S0100-879X2004000900017.

13. Chiu AYY, Au-Ye Ung SSY, LO SK. A comparison of four functional tests in discriminating fallers from non-fallers in older people. Disabil Rehabil. 2003;25:4550. doi:10.1080/713813432.

14. Shumway-Cook A, Baldwin M, Polissar NL, Gruber W. Predicting the probability for falls in community-dwelling older adults. Phys Ther. 1997;77:812-19. PMid:9256869.

15. Berg KO, Wood-Dauphinee SL, Willians JI, Maki B. Measuring balance in the elderly: validation of an instrument. Can J Public Health. 1992;83(Suppl 2):S7-S11. PMid:1468055.

16. Horak FB, Henry SM, Shumway-Cook A. Postural perturbations: new insights for treatment of balance disorders. Phys Ther. 1977;77:517-32.

17. Kauffman TL, Nasher LM, Allison LK. Balance is a critical parameter in orthopedic rehabilitation. New Technol Phys Ther. 1997;6:43-78.

18. Wallace B. Treinamento do equilíbrio. In: Bandy WD, Sanders B. Exercício terapêutico: técnicas para intervenção. Rio de Janeiro: Guanabara Koogan; 2003. p. 229-251.

19. Barbosa SM, Arakaki J, da Silva MF. Estudo do equilíbrio em idosos através da fotogrametria computadorizada. Fisioter. Bras. 2001;2:189-96. 
20. Konrad HR, Girardi M, Helfert R. Balance and Aging. Laryngoscope. 1999;109:454-60. doi:10.1097/ 00005537-199909000-00019.

21. Bretan O, Pinheiro RM, Corrente JE. Avaliação funcional do equilíbrio e da sensibilidade cutânea plantar de idosos moradores na comunidade. Braz. J Otorhinolaryngol. 2010;76:219-24. doi: 10.1590/S1808-86942010000200012.

22. Kaneda K, Sato D, Wakabayashi H, Hanai A, Nomura T. A comparison of the effects of different water exercise programs on balance ability in ederly people. J Aging Phys Act. 2008;16:381-92. PMid:19033600.

23. Resende SM, Rassi CM, Viana FP. Efeitos da hidroterapia na recuperação do equilíbrio e prevenção de quedas em idosas. Rev Bras Fisioter. 2008;12:57-63. doi:10.1590/S1413-35552008000100011.

24. Avelar NCP, Bastone AC, Alcântara MA, Gomes WF. Effectiveness of aquatic and non-aquatic lower limb muscle endurance training in the static and dynamic balance of elderly people. Rev Bras Fisioter. 2010;14:229-36. doi:10.1590/S1413-35552010000300007.

25. Ballard JE, McFarland C, Wallace LS, Holiday DB, Roberson G. The effect of 15 weeks of exercise on balance, leg strength, and reduction in falls in 40 women aged 65 to 89 years. J Am Med Womens Assoc. 2004;59(4):255-61. PMid:16845754.
26. Ikezoe T, Tsutou A, Asakawa Y, Tsuboyama T. Low intensity training for frail elderly women: long term effects on motor function and mobility. J Phys Ther Sci. 2005:17:43-9. doi:10.1589/jpts.17.43.

27. Guimarães LHCT, Geraldino DCA, Martins FLM. Comparação da propensão de quedas entre idosos que praticam atividade física e idosos sedentários. Rev Neurosci. 2004;12:68-72.

28. Coelho CS, Coelho IC. Comparação dos benefícios obtidos através da caminhada e da hidroginástica para a terceira idade. Anais do II Encontro de Educação Física e Áreas Afins Núcleo de Estudo e Pesquisa em Educação Física (NEPEF); 26 e 27 de Outubro de 2007; Piauí, 2007.

29. McDermott AY, Mernitz H. Exercise and older patients: prescribing guidelines. Am Fam physician. 2006;74:437-44.

30. Ransdell LB, Wells CL. Physical activity in urban white, african- american, and mexican-american women. Med Sci Sports Exerc. 1998;30:1608-15.

Recebido: 17/05/2011 Received: 05/17/2011

Aprovado: 03/02/2012

Approved: 02/03/2012 